\title{
TWO-AXIS GIMBALED MICROSCANNER IN DOUBLE SOI LAYERS ACTUATED BY SELF-ALIGNED VERTICAL ELECTROSTATIC COMBDRIVE
}

\author{
Daesung Lee and Olav Solgaard \\ Edward L. Ginzton Laboratory, Department of Electrical Engineering \\ Stanford University, Stanford, CA 94305
}

\begin{abstract}
We present a two-axis, bi-directional gimbaled microscanner in double SOI layers actuated by self-aligned, vertical, electrostatic combdrive actuators. The fabrication process is an extension of our previously developed process for fabrication of single-axis scanners in single-SOI material by adding a noncritical backside etch step. Double-stacked SOI layers are used to provide both electrical isolation and mechanical connections. We demonstrate micromirrors with $\pm 7.5^{\circ}, \pm 7.8^{\circ}$ of static optical deflection on the inner and outer axis, respectively. Resonant frequencies are $3.5 \mathrm{kHz}$ with $\pm 8.8^{\circ}$ optical deflection on the inner axis and $980 \mathrm{~Hz}$ with $\pm 10.5^{\circ}$ optical deflection on the outer axis.
\end{abstract}

\section{INTRODUCTION}

Micromirrors that measure hundreds of micron on a side and provide separately-controllable tilt on two orthogonal axes are required for high-resolution optical MEMS applications like displays, fiber switches [1], and scanning-confocal microscopes [2]. Gimbals actuated by vertical combdrives provide an effective way of implementing decoupled two-axis rotation, and such structures have been demonstrated in polysilicon as well as Silicon-on-Insulator (SOI) with one or two layers of device silicon [3-5]. Designs based on SOI material with two device layers simplify electrical isolation, enabling more complex electrode structures that provide bi-directional operation of both frames. Compared to polysilicon scanners, SOI-based designs also simplify fabrication of mirrors with high optical quality. Gimballess scanners using mechanical rotation transformers have been reported, and have been shown to provide similar scanning speed on both axes [6], which is difficult to achieve with gimbals.

We have recently demonstrated a single-axis micromirror capable of bi-directional rotation and piston motions actuated by self-aligned, vertical, electrostatic combdrives in double-stacked SOI layers $[\mathbf{7 , 8}]$. The fabrication process, which requires only front-side processing, offers three structural layers that consist of upper, lower, and double-stacked layers. Two oxide layers under the device layers provide electrode isolation and etch stops for thickness control. Self-alignment of combs in the two device layers is accomplished by creating masking features in the upper layer and transferring them into the lower layer. These process features can be extended to fabricate two-axis, bi-directional gimbaled microscanners by adding a non-critical backside etch step.

In this paper, we present the design, fabrication process, and experimental results of two-axis, bi-directional gimbaled microscanners in double SOI layers actuated by self-aligned, vertical, electrostatic combdrive actuators.

\section{DESIGN AND FABRICATION}

Figure 1 shows a schematic of the completed two-axis gimbaled microscanner. The mirror, movable combteeth, and inner torsional springs are fabricated in the upper silicon layer. The outer torsional springs are fabricated in double-stacked SOI, while the fixed combteeth are fabricated either in double-stacked SOI as shown in Fig. 1a, or in the lower silicon layer only. A backside etch window is located beneath the gimbaled structure. Four electrodes in the lower layer allow bi-directional rotation of the inner and outer frames. Typically, all parts of the upper layer are grounded, but in some cases two electrodes in the upper layer for the outer frame are connected to two additional actuation voltages. A dual configuration of the mirror can be implemented by changing the role of the upper and lower silicon layer.

(a)
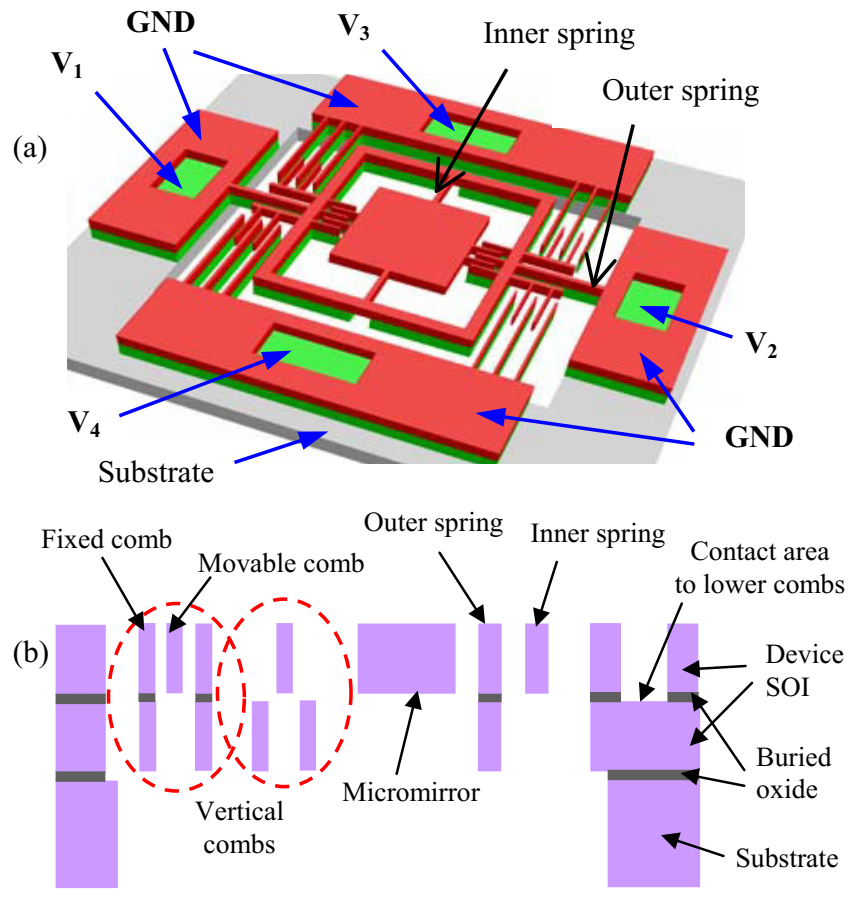

Figure 1. (a) 3D schematic of a two-axis gimbaled micromirror, (b) Cross sectional view of a two-axis gimbaled micromirror

The process starts with DRIE of the device layer (Mask 1) in regular SOI wafers (Fig. 2a). The mask patterns coarse structures encompassing the final dimension of the completed device in the lower device layer with micron scale misalignment margin. The second device layer is created by oxidation, fusion bonding, grinding, and polishing (2b). The two structural layers are both 21 $\mu \mathrm{m}$ thick and the intermittent oxide layer is $0.45 \mu \mathrm{m}$ thick. A single LTO layer $(1.1 \mu \mathrm{m})$ is deposited and patterned by two masks, creating two thickness of LTO; 1.1, $0.4 \mu \mathrm{m}$. The first of the two masks (Mask 2) is the self-alignment mask and patterned into the full thickness of LTO (2c). Then the partial etch of LTO leaving $0.4 \mu \mathrm{m}$ is done with Mask 3, which covers the regions for partial etching with micron scale misalignment margin $(2 \mathrm{~d})$. The

Travel support has been generously provided by the Transducers Research Foundation and by the DARPA MEMS and DARPA BioFlips programs. 
process uses two self-alignment steps; Mask 1 to Mask 2, and Mask 2 to Mask 3. The alignment accuracy needed in each step is $g / 2$ where $g$ is the comb gap. At the completion of the process, these three masks make structures in upper, lower, and doublestacked layers.

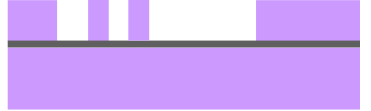

(a) DRIE of coarse patterns (Mask 1)

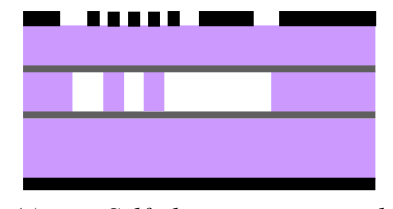

(c) Self-alignment mask patterning (Mask 2) after LTO deposition

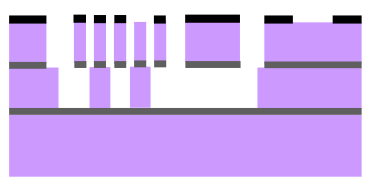

(e) DRIE of the upper device layer followed by directional oxide etch
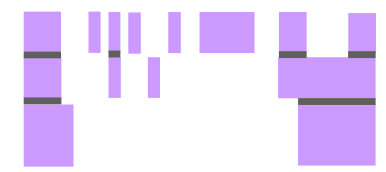

(g) Backside patterning and DRIE followed by directional oxide etch from back and frontside (Mask 5)

Figure 2. Process flow of two-axis gimbaled micromirrors (not to scale)

With the front-side masking with three masks completed, the LTO layer on the backside of the wafer is stripped and alignment marks (Mask 4) are written on the backside of the wafer by etching $0.5 \mu \mathrm{m}$ of silicon. Next, the upper silicon layer is etched and the exposed parts of the oxide layer and remaining thin LTO (initial thickness: $0.4 \mu \mathrm{m}$ ) are directionally etched (2e). Then the lower and upper silicon layers are etched at the same step with silicon and LTO masks patterned by Mask 2 and 3, respectively (2f). Now the front-side etching is completed and the wafer is bonded to an oxidized handle wafer with $1.6 \mu \mathrm{m}$ of photoresist for substrate etching. The backside-etch patterns (Mask 5) are prepared with thick photoresist on the backside of the wafer. Here the previously etched patterns in silicon (Mask 4) are used for alignment.

The silicon substrate $(500 \mu \mathrm{m})$ is then etched by DRIE and the process wafer is separated from the handle wafer by soaking in acetone for 5-7 hours. After being cleaned in Piranha, exposed parts of the oxide layers are directionally etched from the backside. Finally the remaining masking LTO, as well as the exposed intermittent oxide layers are directionally etched from the front-side $(2 \mathrm{~g})$. Figure 3 and 4 show SEM pictures of a finished device with the mirror fabricated in the upper, and lower silicon layer, respectively. In these SEM pictures, fixed combs consist of double-stacked layers. Devices with fixed combs in one layer were successfully fabricated as well. Here small amount of undercut of device layers at the interface of intermittent oxide layers is observed because over etching of silicon is required due to nonuniformity of the etching rate. Large oxide membranes formed under mirrors after the substrate etching, especially when the mirror is fabricated in the upper silicon layer, were observed to crack during the process. However, the structural layers were not affected, and the directional oxide etch from the backside was sufficient to completely remove exposed parts of the oxide layers.

(a)
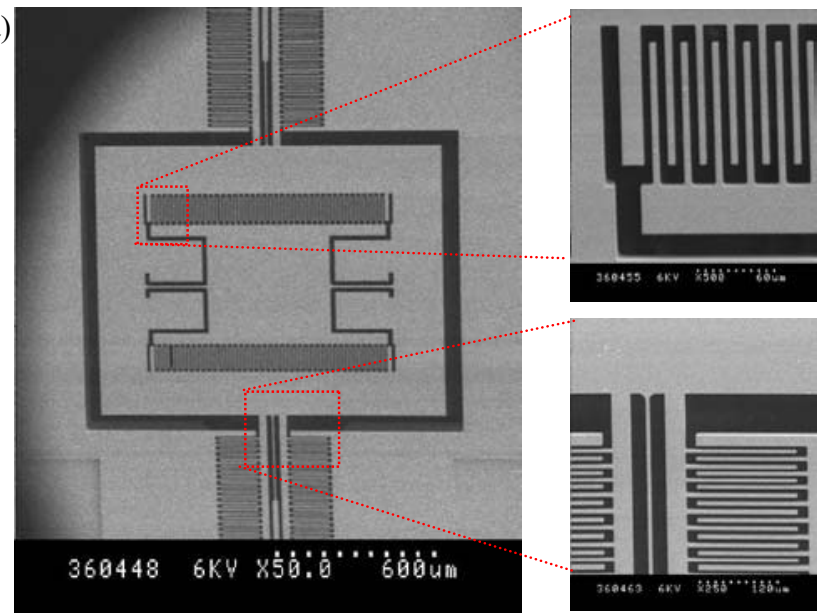

(b)
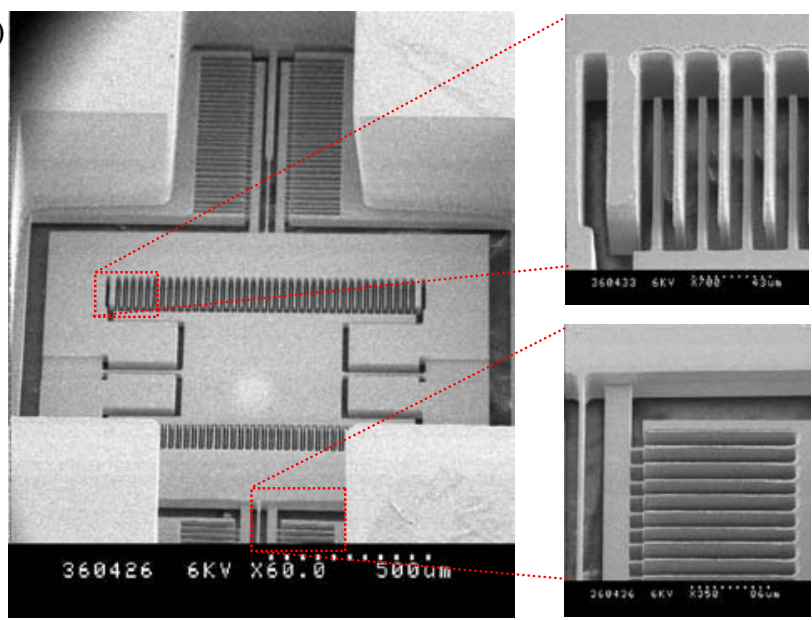

Figure 3. SEM pictures of a two-axis gimbaled micromirror where the mirror is fabricated in the upper silicon layer: (a) Top view, (b) Bottom view

One of the advantages of this process is that isotropic oxide etch is not necessary because directional oxide etch is available both from the front and backside of the wafer. We also observed increased bonding yield by using vacuum bonding to create the second device layer in this process. The sequence of the etch steps is critical. Variations of the process, by switching the order of front and backside etching, have been shown to cause pop-up of mirrors with weak inner torsional springs during the etch of the lower device layer. Double SOI structures after substrate etching were observed to bend, which caused some lateral etching enough to break weak inner torsional springs. This implies that a backside etch window cannot be replaced with a trench fabricated at the 
beginning of the process to provide only front-side processing in double SOI layers.
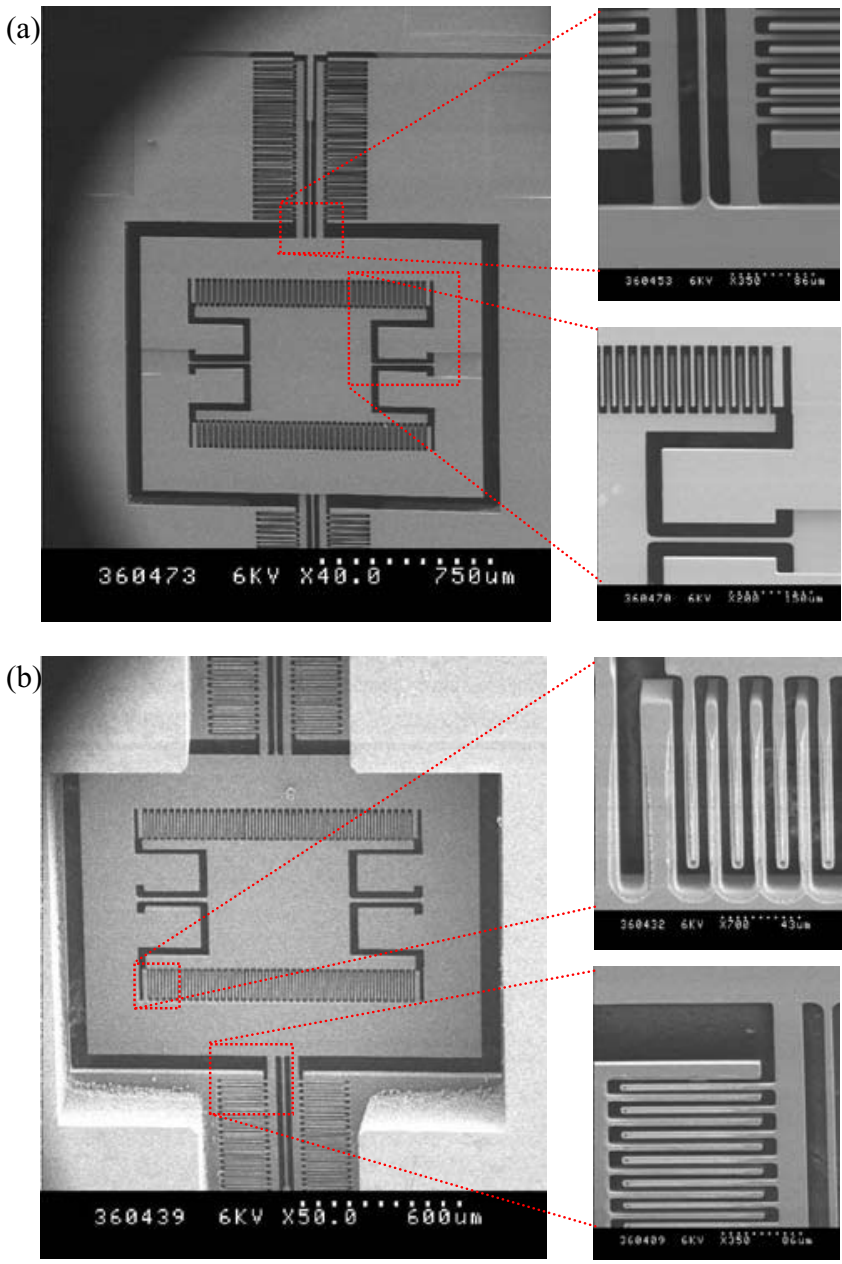

Figure 4. SEM pictures of a two-axis gimbaled micromirror where the mirror is fabricated in the lower silicon layer: (a) Top view, (b) Bottom view

\section{DEVICE CHARACTERIZATION}

Single-crystal-silicon (SCS) mirrors have merits of flat and smooth optical surfaces. In most cases, they are made of a single SOI layer. In our process, the frame and outer torsional springs are made of two SOI layers with an oxide layer in between although mirrors are fabricated in a single SOI layer. The following surface profile measurement and device characterization show that the performance of our devices are close to those of devices made in a single SOI layer.

Figure 5 shows the interferometric image of the frame of a finished device measured by WYKO. The dimension of the frame and micromirror is $1580 \times 1160 \mu \mathrm{m}, 500 \times 500 \mu \mathrm{m}$, respectively. The peak-to-valley surface deformation of the frame is $0.78 \mu \mathrm{m}$ for the horizontal direction and $0.94 \mu \mathrm{m}$ for the vertical direction. The peak-to-valley surface deformation of the micromirror is less than $0.08 \mu \mathrm{m}$. The effect of the stress in the structural layers is mainly the elevation of fixed combs in the inner gimbal, causing the degradation in the performance of vertical combdrives. In our case, the elevation and height variation of fixed combs is less than $0.3 \mu \mathrm{m}$. The elevation of the movable combs in the outer gimbal is less than $0.2 \mu \mathrm{m}$. This shape of surface profile is observed universally across the devices with different spring designs and fabricated from other wafers. The upper and lower silicon layers are $21 \pm 1.5 \mu \mathrm{m}$ thick for all the wafers. The variation of surface deformation of the frame is less than 50 percent.

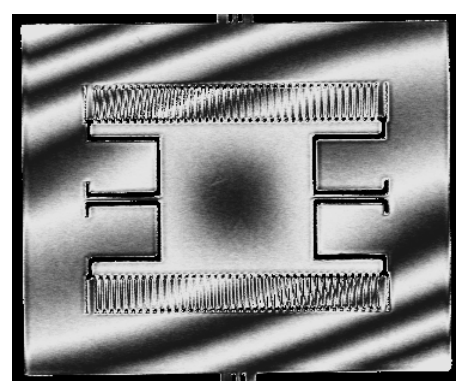

Figure 5. Interferometric surface profile of the frame of the mirror measured by $W Y K O$

The static and dynamic shown in Fig. 6 and 7 are all obtained for mirrors that have torsional springs that measure $250 \times 6 \times 21 \mu \mathrm{m}$ and $400 \times 6 \times 42.5 \mu \mathrm{m}$ for the inner and outer frame, respectively. There are 40 combteeth each measuring $115 \times 6 \times 21 \mu \mathrm{m}$ on the inner frame, and 60 combteeth each measuring $165 \times 6 \times 21 \mu \mathrm{m}$ on the outer frame. The gaps between combteeth are $6 \mu \mathrm{m}$ for both axes. The dimension of the frame is the same as that of the micromirror used for surface profile measurements.

(a)

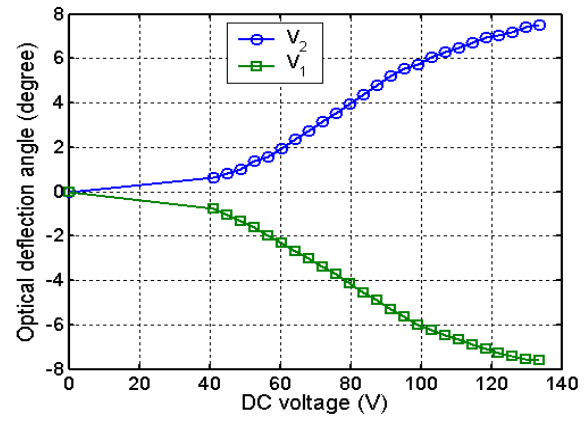

(b)

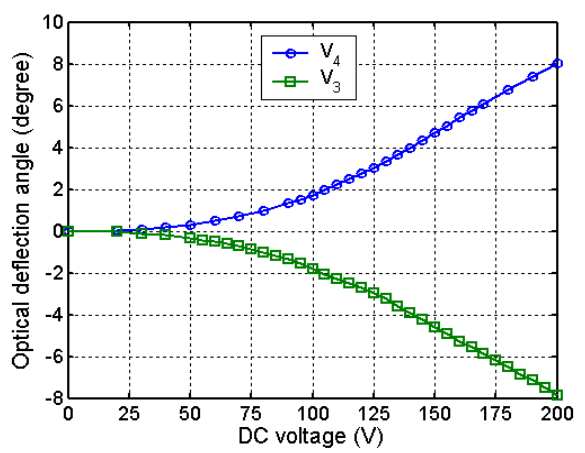

Figure 6. Static optical deflection curves: (a) Inner axis, (b) Outer axis

The measured static optical deflection for a $500 \times 500 \times 21 \mu \mathrm{m}$ microscanner ranged from $-7.6^{\circ}$ at $\mathrm{V}_{1}=133 \mathrm{~V}$ to $7.5^{\circ}$ at $\mathrm{V}_{2}=133 \mathrm{~V}$ on the inner axis (Fig. 6a) and from $-7.8^{\circ}$ at $\mathrm{V}_{3}=200 \mathrm{~V}$ to $8.0^{\circ}$ at $\mathrm{V}_{4}=200 \mathrm{~V}$ on the outer axis (Fig. 6b). The deflection on the inner axis is close to the calculated maximum of $\pm 7.8^{\circ}$ for static operation, while the maximum of $\pm 13^{\circ}$ deflection on the outer axis could not be achieved with practical voltages. 
The observed deflection curves are not quadratic in the voltage because the derivative of the capacitance between combs in the vertical combdrives as a function of rotation is not constant. As the deflection increases the moving combteeth rotate through the full depth of the stationary combteeth, limiting further capacitance increases.

The frequency response was obtained with a driving voltage of $(42+10 \sin \omega t) \mathrm{V}$ on $\mathrm{V}_{2}$ for the inner axis (Fig. 7a), and on $\mathrm{V}_{4}$ for the outer axis (Fig. 7b). The resonant frequency was $3.5 \mathrm{kHz}$ with $\pm 8.8^{\circ}$ optical deflection on the inner axis and $980 \mathrm{~Hz}$ with $\pm 10.5^{\circ}$ optical deflection on the outer axis.

The data was collected by measuring the output waveform of a PSD (position sensitive detector) whose input is the reflected beam from the mirror surface. On both axes, three major peaks were observed. The driving frequencies at these peaks are measured to be $\omega_{0} / 2, \omega_{0}$, and $2 \omega_{0}$ where $\omega_{0}$ is the resonant frequency of the rotational mode. At each of these peaks, the mirror was observed to rotate at the resonant frequency. In addition to the major peaks, we also observed minor peaks at $1 / 3$, $1 / 4$, and $1 / 5$ of the resonant frequency. At these peaks, the rotation was a combination rotation at the driving and resonant frequencies. The peaks below the fundamental resonance are due to the nonlinear relationship between voltage and electrostatic force.

(a)

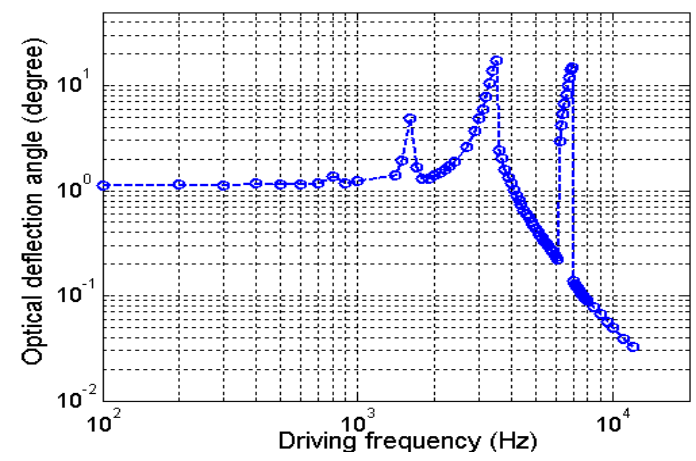

(b)

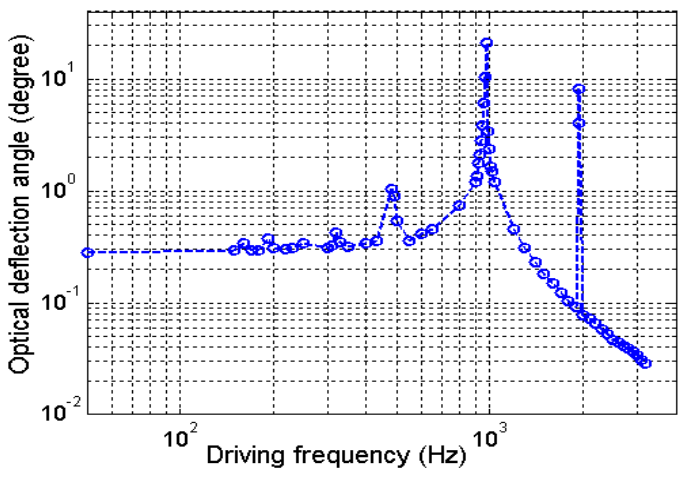

Figure 7. Frequency response: (a) Inner axis, (b) Outer axis

At the peak at $2 \omega_{0}$, the mirror rotates at the resonant frequency, i.e. half the driving frequency. The likely cause is that large parts of the moving combs protrude on the opposite side of the stationary combs when the moving comb rotates through large angles. Under such large angular deflection, the electrostatic force is therefore directed oppositely to its direction under small rotation. The effect of this "backward" electrostatic force is that a drive signal at twice the resonant frequency can give rise to motion at the resonance as observed.

\section{CONCLUSIONS}

We have demonstrated two-axis, bi-directional gimbaled microscanners fabricated in SOI material with two devices layers. The fabrication process requires 5 lithography steps, and includes self-alignment and etch stops of all critical etch steps. The two isolated devices layers enable bi-directional operation on both axes while maintaining good surface profiles. We demonstrated static optical rotation of more than $\pm 7.5^{\circ}$ on the inner axis and $\pm 7.8^{\circ}$ on the outer axis of gimbaled scanning mirrors measuring $500 \times 500 \times 21 \mu \mathrm{m}$. On resonance, the achieved scan angles are \pm $8.8^{\circ}$ and $\pm 10.5^{\circ}$ on the inner and outer axis, respectively.

We expect that this type of gimbaled microscanners as well as this process technology will find a variety of applications including displays, scanning-confocal microscopes, wave-front correctors, and optical fiber switches.

\section{ACKNOWLEDGEMENT}

The authors gratefully acknowledge support from the Defense Advanced Research Projects Agency under contract (DAAH01-00C-0089).

\section{REFERENCES}

1. R. Ryf, et al, "1296-port MEMS Transparent Optical Crossconnect with 2.07 Petabit/s Switch Capacity", Technical Digest of Optical Fiber Communication Conference 2001, Anaheim, CA, USA, Mar. 2001, Post deadline paper PD-28.

2. D.L. Dickensheets, G.S. Kino, "Silicon-Micromachined Scanning Confocal Optical Microscope", Journal of Microelectromechanical Systems, vol. 7, no. 1, Mar. 1998.

3. S. Kwon, V. Milanovic, L. P. Lee, "A High Aspect Ratio 2D Gimbaled Microscanner with Large Static Rotation”, 2002 IEEE/LEOS Int. Conf. on Optical MEMS, Lugano, Switzerland, Aug. 2002.

4. N. Kouma, O. Tsuboi, Y. Mizuno, H. Okuda, X. Mi, M. Iwaki, H. Soneda, S. Ueda, I. Sawaki, "A Multi-Step DRIE Process for A 128 x 128 Micromirror Array", 2003 IEEE/LEOS Int. Conf. on Optical MEMS, Hawaii, USA, Aug. 2003.

5. D. Han, C.-A. Choi, C.-H. Jun, Y. T. Kim, "A Self-Aligned Vertical Comb-Drive Actuator Using Surface Micromachining for Scanning Micromirrors", 2003 IEEE/LEOS Int. Conf. on Optical MEMS, Hawaii, USA, Aug. 2003.

6. V. Milanovic, G. Matus, T. Cheng, B. Cagdaser, "Monolithic High Aspect Ratio Two-axis Optical Scanners in SOI", Int. Conf. on Microelectromechanical Systems, MEMS2003, Kyoto, Japan, Jan. 2003.

7. D. Lee, U. Krishnamoorthy, K. Yu, O. Solgaard, "HighResolution, High-Speed Microscanner in Single-Crystalline Silicon Actuated by Self-Aligned Dual-Mode Vertical Electrostatic Combdrive with Capability for Phased Array Operation", Transducers '03, Boston, USA, Jun. 2003.

8. D. Lee, U. Krishnamoorthy, K. Yu, O. Solgaard, "SingleCrystalline Silicon Micromirrors Actuated by Self-Aligned Vertical Electrostatic Combdrives with Piston-motion and Rotation Capability", submitted to Sensors and Actuators: A (Physical), Jul. 2003 (accepted for publication) 